\title{
Selective Interaction of Moving Neutral Particles with Modulated Surface and the Mechanism of Controlled Surface Nanofabrication*
}

\author{
Vladimir I. Vysotskii, ${ }^{\dagger}$ Mykhaylo V. Vysotskyy, and Nikolae V. Maksyuta \\ Taras Shevchenko National University of Kyiv, Radio Physics Faculty, Kyiv, Ukraine \\ (Received 12 January 2014; Accepted 31 May 2014; Published 9 August 2014)
}

\begin{abstract}
It is shown that neutral particles moving above modulated surfaces undergo selective attractive or repulsive forces which in a resonance way depend on transition frequencies between internal energetic levels and the velocity of atom motion. The interaction of hydrogen atoms with surface-inhomogeneous periodic electric fields of crystallographic planes of ionic and ionic-covalent crystals is studied. It is investigated the possibility of application of such interactions for the obtaining of monochromatic by velocities one-component atom beams and also for the classification and separation of multi-component beams of particles for the determination the degree of ionicity, etc. [DOI: 10.1380/ejssnt.2014.387]
\end{abstract}

Keywords: Atom-solid interactions; Surface structure; Hydrogen atom; Channeling; Processing and fabrication; Ionic crystal; Ionic-covalent crystal; Degree of ionicity

\section{INTRODUCTION}

The process of neutral particles interaction with internal electromagnetic structure (for example, atoms) moving nearby solid bodies surfaces beside which there exist natural or artificially fabricated periodic electric fields is determined by specific resonance forces. Such fields, for example, exist quite close to a domain system in electrets or ionic crystals. They also may be originated at ferroelectrics ultrasound modulation.

The nature of these forces is close to those ones which appear in the result of inhomogeneous electromagnetic radiation effect on moving atoms (see, for example, [1]). Really, in an attendant particle coordinate system a static periodic electric field (with $\Lambda$ period) corresponds to oscillating in time electric field of pseudo-photons stream (see [2]) with so-called bounce-frequencies $\Omega_{n}=2 \pi n v / \Lambda$, where $n=1,2, \ldots, \boldsymbol{v}=v \boldsymbol{e}_{z}$ - a neutral particle motion velocity. The coincidence of one of these frequencies (for example, $\Omega_{1}=2 \pi v / \Lambda \equiv \Omega$ at $n=1$ ) with the frequency $\omega_{21}$ of the internal resonance transition between the arbitrary quantum levels of 1 and 2 leads to the interaction between the induced in a particle electric dipole moment and an electric by-surface field forming this moment. In [3] studying mesoatoms volumetric channeling at the expense of the interaction of crystallographic planes with periodic fields such interaction was called an induceddispersive one.

Below, in the frames of density matrix formalism it has been got a general expression for an average energy interaction of moving neutral particles with surfaceinhomogeneous periodic electric fields and concrete examples analyze a hydrogen atoms motion along electroneutral and charged crystallographic planes of ionic and ionic-covalent crystals.

\footnotetext{
* This paper was presented at the 12th International Conference on Atomically Controlled Surfaces, Interfaces and Nanostructures (ACSIN-12) in conjunction with the 21st International Colloquium on Scanning Probe Microscopy (ICSPM21), Tsukuba International Congress Center, Tsukuba, Japan, 4-8 November, 2013.

† Corresponding author: vivysotskii@gmail.com
}

\section{THE CALCULATION OF AN AVERAGE ENERGY INTERACTION OF MOVING NEUTRAL PARTICLE WITH A MODULATED SURFACE}

Let's consider a special case when a moving neutral particle undergoes the action of two electric fields: a constant electric field with $\boldsymbol{E}_{0}=E_{0} \boldsymbol{e}_{x}$ tension and surfaceinhomogeneous periodic electric field of the type of

$$
\boldsymbol{E}(x, z)=E_{k} \exp (-k x)\left[\boldsymbol{e}_{x} \cos (k z)+\boldsymbol{e}_{z} \sin (k z)\right],
$$

where $k=2 \pi / \Lambda$. Further calculations will be done in coordinate system moving along $z$ axis. In this system the field (1) according to $z=z^{\prime}-v t^{\prime}, x=x^{\prime}$, $t=t^{\prime}$ Galileo transformations (particle motion is nonrelativistic) is presented in the type of

$$
\begin{aligned}
& \boldsymbol{E}\left(x^{\prime}, z^{\prime}, t^{\prime}\right)=E \exp \left(-k x^{\prime}\right) \\
& \quad \times\left[\boldsymbol{e}_{x} \cos \left(k z^{\prime}-\Omega t^{\prime}\right)+\boldsymbol{e}_{z} \sin \left(k z^{\prime}-\Omega t^{\prime}\right)\right] .
\end{aligned}
$$

In Fig. 1 it is schematically shown this field polarization alteration on one period and also the place of a neutral atom in an attendant coordinate system (further hatches are not written down). Thus, if a neutral particle is in $(x, 0,0)$ point (see Fig. 1$)$, then a composite electric field affecting it in an attendant coordinate system is written down in the form

$$
\begin{aligned}
\boldsymbol{E}(x, t)= & \boldsymbol{e}_{x} E_{0}+E_{k} \exp (-k x) \\
& \times\left[\boldsymbol{e}_{x} \cos (\Omega t)-\boldsymbol{e}_{z} \sin (\Omega t)\right] .
\end{aligned}
$$

Besides, an instantaneous value of an operator of a dipole interaction with the field (3), written down in complex variables is described by the expression

$$
\begin{aligned}
V(x \boldsymbol{\xi}, t) & =-\boldsymbol{d}(\boldsymbol{\xi}) \boldsymbol{E}(x, t) \\
& =V_{0}(\boldsymbol{\xi})+V_{k}(x \boldsymbol{\xi}) \exp (-i \Omega t) .
\end{aligned}
$$

Here $\boldsymbol{d}(\boldsymbol{\xi})=-e \boldsymbol{\xi}$ - an operator of an atom dipole momentum being a function of coordinates $\boldsymbol{\xi}$ totality, describing an atom internal motion

$$
\begin{aligned}
U_{0}(\boldsymbol{\xi}) & =-d_{x}(\boldsymbol{\xi}) E_{0} \\
U_{k}(x \boldsymbol{\xi}) & =-E_{k} \exp (-k x)\left[d_{x}(\boldsymbol{\xi})-i d_{z}(\boldsymbol{\xi})\right] / 2 .
\end{aligned}
$$




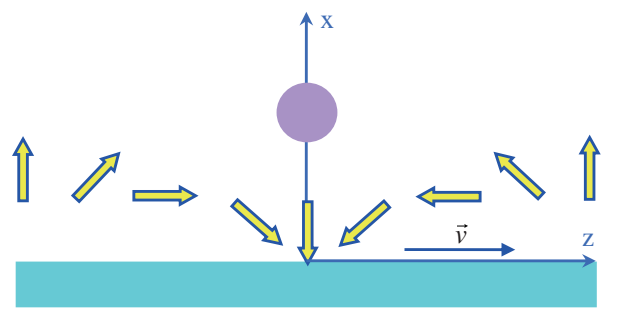

FIG. 1. Schematic interpretation of the electric field polarization alteration on one period and the place of a neutral atom in the attendant coordinate system.

Then substitute the expression (4) in the equation for a matrix density $\rho(x \boldsymbol{\xi}, t)$

$$
\begin{aligned}
i \hbar \frac{\partial \rho(x \boldsymbol{\xi}, t)}{\partial t}=\left\{H_{0}(\boldsymbol{\xi})\right. & +U(x \boldsymbol{\xi}, t) \rho(x \boldsymbol{\xi}, t)\} \\
& -i \hbar \Gamma \rho(x \boldsymbol{\xi}, t),
\end{aligned}
$$

where $H_{0}(\boldsymbol{\xi})$ - a Hamilton operator describing a neutral atom internal motion, $\Gamma \rho$ - a phenomenological given term, taking into account matrix density relaxation elements; diagonal elements $(\Gamma \rho)_{n n}=\left(\rho_{n n}-\rho_{n n}^{(0)}\right) / T_{1}$ describe level population, fading during the longitudinal relaxation $T_{1}$; non-diagonal elements $(\Gamma \rho)_{m n}=\rho_{m n} / T_{2}$ describe a high-frequency dipole moment, fading during the transverse relaxation $T_{2}$ (here $m, n=1,2, \rho_{n n}^{(0)}$ - an equilibrium probability of the level $n$ population).

Suppose that relaxation fulfills only at the expense of spontaneous transitions that correspond to the equality $T_{1}=T_{2}=\gamma^{-1}[4]$. To simplify further analysis let's take $\rho_{11}^{(0)}=1, \rho_{22}^{(0)}=0$.

Using the distribution

$$
\rho(x \boldsymbol{\xi}, t)=\sum_{\alpha, \beta=1}^{2} \rho_{\alpha \beta}(x, t) \Psi_{\alpha}(\boldsymbol{\xi}) \Psi_{\beta}^{*}(\boldsymbol{\xi})
$$

of the density matrix $\rho(x \boldsymbol{\xi}, t)$ by proper functions $\Psi_{\alpha}(\boldsymbol{\xi}, t)=\psi_{\alpha}(\boldsymbol{\xi}) \exp \left(-i \epsilon_{\alpha} t / \hbar\right)$ of the Hamiltonian $H_{0}(\boldsymbol{\xi})$, we pass from the equation (5) to the following equation system for $\rho_{\alpha \beta}(x, t)(\alpha, \beta=1,2)$ :

$$
\begin{aligned}
\frac{\partial \rho_{22}}{\partial t} & =\frac{1}{i \hbar}\left(V_{21} \rho_{12}-\rho_{21} V_{12}\right)-\gamma \rho_{22}, \\
\frac{\partial \rho_{21}}{\partial t} & =\frac{V_{21}\left(\rho_{11}-\rho_{22}\right)}{i \hbar} \\
& +\left(\frac{V_{22}-V_{11}}{i \hbar}-i \omega_{21}-\gamma\right) \rho_{21},
\end{aligned}
$$

where $V_{11,22}=\left(U_{0}\right)_{11,22}, V_{12}(x, t)=V_{21}^{*}(x, t)=$ $V_{12}(x) \exp [-i(\Omega t+\pi / 2)]-$ diagonal and non-diagonal matrix elements of the dipole interaction operators, $V_{12}(x)=\left(U_{k}(x)\right)_{12}=-E_{k} d \exp (-k x)$ (here the equality $\left(d_{z}\right)_{12}=i\left(d_{x}\right)_{12}=d$, which is checked in the following item on the concrete example), $\rho_{12}=\rho_{21}^{*}, \rho_{11}=1-\rho_{22}$.

Using the substitutions of

$$
\begin{aligned}
& \rho_{21}=\sigma_{21} \exp [-i(\Omega t+\pi / 2)], \\
& \rho_{12}=\rho_{21}^{*}=\sigma_{12} \exp [i(\Omega t+\pi / 2)],
\end{aligned}
$$

in the stationary regime $\left(d \sigma_{\alpha \beta} / d t=0\right)$ we find the solution of the equation system (6):

$$
\begin{gathered}
\rho_{22}=\frac{2 g^{2}}{\Delta \omega^{2}+\gamma^{2}+4 g^{2}}, \rho_{11}=1-\rho_{22}, \\
\rho_{21}=\rho_{12}^{*}=\frac{g(\Delta \omega-i \gamma)}{\Delta \omega^{2}+\gamma^{2}+4 g^{2}} \exp (-i(\Omega t+\pi / 2)),
\end{gathered}
$$

where $\Delta \omega=\Omega-\omega_{21}+g_{11}-g_{22}=\Delta \omega^{(0)}+g_{11}-g_{22}$ a frequency breaking, $g(x)=V_{12}(x) / \hbar, g_{11,22}=V_{11,22} / \hbar$.

At last, by means of the expressions (7) and matrix elements values of $V_{11,22}$ and $V_{12,21}(x, t)$ we find an average energy interaction of moving atom with an electric field (3) $[1]$ :

$$
\begin{aligned}
\langle V(x)\rangle & =\sum_{\alpha, \beta=1}^{2} V_{\alpha \beta} \rho_{\beta \alpha} \\
& =\frac{\hbar G(x) \Delta \omega^{(0)}}{2\left[1+G(x)+(\Delta \omega / \gamma)^{2}\right]}+\hbar g_{11},
\end{aligned}
$$

where $G(x)=(2 g(x) / \gamma)^{2}$ - a saturation parameter [1]

From the formula (8) it is seen that depending on sign of the value $\Delta \omega^{(0)}$ an atom repulses (for "+") or attracts (for "-") to a surface. The further analysis of the dependence (8) we'll carry out on a concrete example.

\section{RESONANCE INTERACTION OF HYDROGEN ATOM WITH}

\section{CRYSTALLOGRAPHIC PLANES OF IONIC AND} IONIC-COVALENT CRYSTALS

A particle moving along the crystallographic plane of ionic or ionic-covalent crystal is effected by periodically arranged positively and negatively charged ions of the lattice, which one-particle potentials are written in the form

$$
\phi_{ \pm}(r)=\phi_{A^{+}, B^{-}}(r) \pm \frac{\alpha e}{r}
$$

where $\alpha$ - ionicity degree of crystal atoms, $\phi_{A^{+}, B^{-}}(r)$ - one-particle potentials of neutral stems of $A^{+}$and $B^{-}$ ions of considerable respectively.

Obviously, the main contribution to the periodic electric fields of such planes at distances larger than atomic shielding radii gives only the second components in the formula (9). Proceeding from this, we may consider two situations.

a) The first situation occurs when a neutral particle moving near the planes built from ions of different signs (for example, these are the planes (100) in ionic crystals of sodium chloride type [5]). In this case, the calculation of the electric field tension using two-dimensional vectors procedure of the expansion of the reciprocal lattice (for example, see [6]) for these planes leads to the following expression:

$$
\boldsymbol{E}(x, \boldsymbol{\rho})=\frac{8 \pi \alpha e}{a^{2}} \sum_{\boldsymbol{\kappa}}\left(\boldsymbol{e}_{x}-\frac{i \boldsymbol{\kappa}}{\kappa}\right) \exp (i \boldsymbol{\kappa} \boldsymbol{\rho}-\kappa|x|),
$$

where $\boldsymbol{\kappa}=\boldsymbol{\kappa}_{m n}=[0,2 \pi(2 m+1) / a, 2 \pi(2 n+1) / a]-\mathrm{a}$ two-dimensional vector of the reciprocal lattice, $m, n=$ $0, \pm 1, \ldots, a$ - the lattice crystal constant. If the velocity 


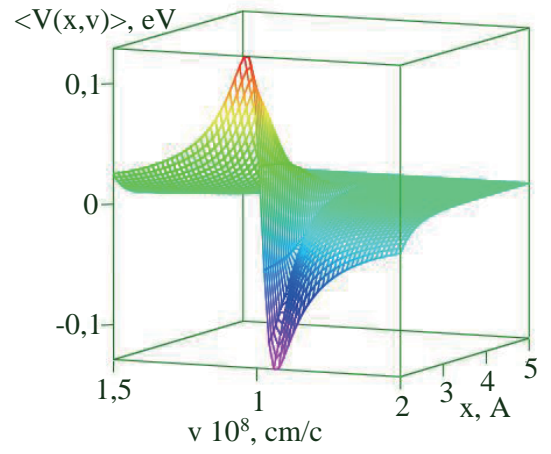

FIG. 2. Graph of an average interaction energy $\langle V(x, v)\rangle$ of the moving hydrogen atom with the crystallographic planes (100) of an ionic crystal RbF.

of the moving particle is collinear, for example, one of the reciprocal lattice vectors of $\boldsymbol{\kappa}_{m n}$, then, as it can be seen from formula (10), the electric field tension for the corresponding component in the associated coordinate system is described by the formula (2). Next, consider the case when the main contribution to the dipole interaction in the expansion (10) makes the component 00 for which the bounce frequency $\Omega=v \kappa_{00}=2 \sqrt{2} \pi v / a$ is close to the transition frequency of $\omega_{21}$. Obviously, in this case, the constant component of the electric field $\boldsymbol{E}_{0}$ is absent, $E_{k}=16 \pi \alpha e / a^{2}$, and the average energy of the interaction, as a function of variables $x$ and $v$, can be rewritten as

$$
\langle V(x, v)\rangle=\frac{\hbar G(x) \Delta \omega^{(0)}(v)}{2\left\{1+G(x)+\left[\Delta \omega^{(0)}(v) / \gamma\right]^{2}\right\}} .
$$

We now calculate the meanings of the values in the formula (11) in the case of the motion of the hydrogen atom over the surface of an ionic crystal. Transition frequency between the ground state with the wave function $\psi_{1} \equiv \psi_{100}(r)$ and the first excited state with the wave function $\psi_{2} \equiv \psi_{21,-1}(r, \theta, \phi)$ is equal to $\omega_{21}=$ $\left(\epsilon_{2}-\epsilon_{1}\right) / \hbar=3 e^{2} / 8 \hbar a_{0}$, where $a_{0}-$ the Bohr radius. This result is related to the fact that in the attendant coordinate system $x z$ the electric field vector rotates in the clockwise direction, the quantization axis is $y$ axis and the nonzero dipole transitions are possible only when the wave function $\psi_{21,-1}(r, \theta, \phi)$ is used. Dipole moment for this transition is equal to $\boldsymbol{d}_{21}=d\left(\boldsymbol{e}_{z}+i \boldsymbol{e}_{x}\right)$ value, where $d=-2^{7} e a_{0} / 3^{5}$. Accordingly, the total probability of spontaneous transition between the levels of $\epsilon_{2}$ and $\epsilon_{1}$ is equal to $\gamma=4 \boldsymbol{d}_{21}^{2} \omega_{21}^{3} / 3 \hbar c^{3}[1]$. Substituting these values into the formula (11) and choosing an ionic crystal with a sufficiently large value of the ionicity degree $\alpha \approx 0.96$ (see, for example, the crystal RbF [7]), we obtain the graph of the function $\langle V(x, v)\rangle$ shown in Fig. 2 .

b) Another situation arises at neutral atoms motion near charged crystallographic planes (for example, (100) planes in crystals with a blende structure [5]) constructed of ions of one kind. In this case the presence of a constant component $\boldsymbol{E}_{0}=\boldsymbol{e}_{x} 4 \pi \alpha e / a^{2}$ leads to the Stark splitting of the excited level $\epsilon_{2}$ of the hydrogen atom on three sublevels with the energies $\epsilon_{2}^{(0)}=\epsilon_{2}, \epsilon_{2}^{( \pm)}=\epsilon_{2} \pm 3 e a_{0} E_{0}$.
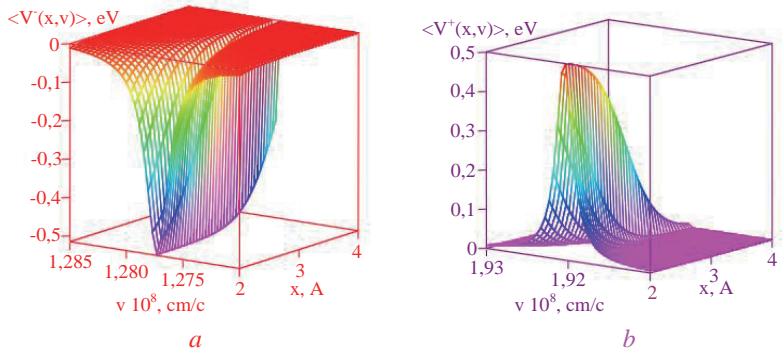

FIG. 3. Graphs of the average energy of interaction of the moving hydrogen atom with crystallographic planes (100) of ioniccovalent crystal InSb: a) for $\left\langle V^{-}(x, v)\right\rangle$, b) for $\left\langle V^{+}(x, v)\right\rangle$.

Nonzero matrix elements of the dipole moment $\boldsymbol{d}(\boldsymbol{\xi})$ exist only on the transitions $\epsilon_{2}^{( \pm)} \rightarrow \epsilon_{1}$ and are equal to $\boldsymbol{d}_{21}^{( \pm)}=\left(2^{6} e a_{0} / 3^{7}\right) \boldsymbol{e}_{x}$. This result is determined by taking into account that the levels $\epsilon_{2}^{( \pm)}$correspond to the wave functions $\psi_{2}^{( \pm)}=\left(\psi_{200} \mp \psi_{210}\right) / \sqrt{2}$ and as the quantization axis the axis $x$ is chosen.

Accounting the above expressions of $E_{k}=8 \pi \alpha e / a^{2}$, $\boldsymbol{d}_{11}=0, \boldsymbol{d}_{22}^{( \pm)}=\mp 3 e a_{0} \boldsymbol{e}_{x}$, and $\gamma^{( \pm)}=4 \boldsymbol{d}_{21}^{2}\left(\omega_{21}^{( \pm)}\right)^{3} / 3 \hbar c^{3}$ $\left(\omega_{21}^{( \pm)}=\left(\epsilon_{2}^{( \pm)}-\epsilon_{1}\right) / \hbar\right)$, in Fig. 3a, b the graphs of the dependencies $\left\langle V^{( \pm)}(x, v)\right\rangle$ are plotted as calculated using the formula (8) for the hydrogen atom moving along (100) planes of ion-covalent crystal InSb with a small value of the ionicity degree $\alpha \approx 0.15$ (see [8]). Note that the choice of this value $\alpha$ is dictated by the requirement of reducing the "Stark" level splitting $\epsilon_{2}$.

\section{CONCLUSIONS}

The given results imply that the interaction potentials of moving hydrogen atoms with a spatially inhomogeneous periodic electric fields of ionic and ionic-covalent crystals at distances, comparing with lattice constants, are quite strong and are characterized by narrow resonances at certain velocities of particles motion. In particular, in the absence of a permanent component the potential character (i.e. the transition from repulsion to attraction) varies at one particular velocity (see Fig. 2). In the presence of such constant electric field component and due to splitting of the excited level, resonant character of the average energy of the hydrogen atom interaction with charged planes ionic-covalent crystals is shown at high velocities. In particular, in one narrow range of velocities, there is quite strong resonance attraction (see Fig. 3a), and another (shifted by the amount of $\left.v^{( \pm)} \approx 4\left(\omega_{21}^{(+)}-\omega_{21}^{(-)}\right) / k\right)$ a narrow range of velocities a strong repulsion resonance presents (see Fig. $3 \mathrm{~b}$ ).

It is obvious that similar resonant effects will occur just at the motion of other neutral atoms in the modulated electric fields (e.g., using a strong electric field electrets [9]). This means that such interactions allow to carry out quite an effective monochromatization by velocities of one-component atomic beams, fabricate sorting and separation of multi-particle beams, to use particle beams for the study of crystal surfaces (in particular, with the help 
of such resonance potentials we can determine the ionicity degrees of ionic and ionic-covalent crystals), etc.
As shown in $[10,11]$, these potentials can contribute to surface and volume channeling of neutral particles and, hence, additional control of particles motion.
[1] V. G. Minogin and V. S. Letokhov, Pressure of Laser Radiation on Atoms (Nauka, Moscow, 1986).

[2] J. Jackson, Classical Electrodynamics (John Wiley \& Sons, 1962).

[3] V. I. Vysotskii and N. V. Maksyuta, Induced Dispersion Mechanism in Oriented Motion of Neutral Particles in Crystals, Preprint IMF 13-90. Kiev: 1990, p. 17.

[4] P. G. Krukov and V. S. Letokhov, Sov. Phys. Usp. 99 , 169 (1969)

[5] N. Ashkroft and N. Mermin, Solid State Physics (Harcourt, 1976).

[6] Y.-K. Otsuki, Charged Beam Interaction with Solids (Mir,
Moscow, 1985).

[7] C. Kittel, Introduction to Solid State Physics (John Wiley \& Sons, 1971).

[8] S. S. Batsanov, Structural Chemistry (facts and dependences) (Dialog - MSU, Moscow, 2000).

[9] F. N. Gubin, Electrets (Nauka, Moscow, 1978).

[10] V. I. Vysotskii and R. N. Kuzmin, Sov. Phys. Usp. 35, 725 (1992).

[11] V. I. Vysotskii, M. V. Vysotskyy, and N. V. Maksyuta, J. Surf. Invest.-X-Ray+ 4, 696 (2010). 